\title{
EVALUACIÓN DE TECNOLOGÍAS DE COMUNICACIÓN PARA REDES VEHICULARES DE ÚLTIMA GENERACIÓN
}

\section{COMMUNICATION-TECHNOLOGY EVALUATION FOR LA- TEST-GENERATION VEHICULAR NETWORKS}

\begin{abstract}
The increasing number of motor vehicles such as cars, buses and lorries; together with the lack of road planning and design in the past have caused serious problems in terms of congestion, accident rates, insecurity, and available information for users of the transport system. Governments and decentralized institutions have been designing communication systems between vehicles with either WAN or Internet access, allowing drivers and passengers to have real-time information about road conditions, tolls, optimal routes and even cultural information about the area, which makes the trip safer and more enjoyable. This paper describes the current state of wireless technologies for communication on next-generation vehicle networks. A comparison is made between the technologies used inside and outside vehicles. The article also presents state-of-the-art architectures employed in Europe and the United States, which are still under consideration and implementation.
\end{abstract}

Keywords: V2V, V2I, WIMAX, WIFI, RFID, ZIGBEE, CALM

\section{Resumen}

El incremento del número de vehículos terrestres particulares, de carga y transporte masivo, y la ausencia de planificación y proyección de las vías en el pasado, han generado graves problemas de congestión, aumento de la accidentalidad, inseguridad y desinformación de los usuarios de este tipo de transporte.

Los gobiernos y las entidades descentralizadas han venido diseñando sistemas de comunicación entre vehículos con acceso a redes Wan o Internet, que permiten a conductores y pasajeros obtener información en tiempo real acerca del estado de las vías, peajes, rutas optimas e incluso acceso a material informativo de la zona por la que se transita, para hacer el viaje mas seguro y placentero.

En este trabajo se describe el estado actual de las tecnologías inalámbricas para la comunicación de las redes vehiculares de última generación, haciendo comparativos entre las tecnologías empleadas a bordo del vehículo y fuera de este. También se muestra el estado del arte en las principales arquitecturas diseñadas en Europa y Estados unidos, que se encuentran en fase de estudio e implementación.

Palabras clave: V2V, V2I, WIMAX, WIFI, RFID, ZIGBEE, CALM

\section{Jorge E. Florez}

Ingeniero de Sistemas, Especialista en Teleinformática. Ingeniero de Soporte en la empresa Quik LTDA.

Bogotá, Colombia.

jorge_florez@quikltda.com

\section{José A. Rojas}

Licenciado en Electrónica, Especialista en Teleinformática.

Operación en Centro de Gestión Integral, COVIANDES.

Bogotá, Colombia.

Lic_jarg@yahoo.es

Danilo A. López.

Ingeniero Electrónico, MSc. en Teleinformática, Docente de planta de la Universidad Distrital "Francisco José de Caldas". dalopez@udistrital.edu.co

Tipo: Artículo de reflexión

Fecha de Recepción: Abril 12 de 2010 Fecha de Aceptación: Mayo 14 de 2010 


\section{INTRODUCCIÓN}

Los Sistemas Inteligentes de transporte (ITS) y más concretamente los servicios en el entorno del automóvil, también conocidos como vehiculares, están experimentando un gran auge en los últimos años. La optimización del transporte por carretera, haciéndolo mas seguro, eficiente y sostenible se ha convertido en una prioridad para las autoridades, la industria y la comunidad científica.

Resolver los problemas de movilidad de forma eficiente y sostenible es uno de los grandes retos de la sociedad actual. El vehículo es el tercer escenario tras el hogar y la oficina donde un ciudadano medio pasa más tiempo. A pesar de esto, existen numerosos factores susceptibles de mejora aplicando servicios de telecomunicaciones. Algunos de los más importantes son:

- Accidentes, emergencias en ruta, averias.

- Trancones e incidentes en la vía.

- Problemas de estacionamiento.

- Desconocimiento de ruta (optima) o posición.

- Viajes aburridos, tiempo perdido en el automóvil.

Estos inconvenientes suponen una oportunidad para el despliegue de servicios TIC. La diversidad de servicios susceptibles de ser desplegados en el entorno del carro ha provocado que tanto la industria como comunidad científica trabaje actualmente de forma intensa en comunicaciones entre vehículo (V2V) y entre estos y la infraestructura (V2I). Tales servicios se pueden clasificar en cuatro grandes bloques:

- Seguridad vial: Aquellos servicios destinados a mejorar la seguridad, evitando accidentes o disminuyendo sus consecuencias si ya se han producido. Ejemplos típicos de esta categoría son el envío de alertas tras detectar un peligro en la calzada, o el servicio eCALL que tras un accidente transmite a un centro de emergencias, toda la información disponible sobre el mismo, a la vez que establece una comunicación vocal.

- Eficiencia en el tráfico: Aquellos servicios orientados a optimizar el uso de las vías, evitando situaciones de congestión y facilitando el acceso a los carros de emergencias.

- Infotainment: Relacionados con la información y el entretenimiento para conductor y pasajeros, como reproducción de contenidos multimedia, acceso a noticias, servicios de navegación avanzada, servicios ofimaticos, comparticion de ruta, redes sociales en el vehículo, etc.

- M2M: Esta categoría engloba aquellos servicios en los que su funcionamiento no implica la interacción humana, como los relacionados con el pago de impuestos o seguros dependiendo del uso (conocidos como "Pay as you drive") monitorizando la forma de conducción y la huella ecológica de los conductores, la gestión de flotas, el diagnostico remoto, sistemas antirrobo y localización, control de la documentación electrónica del vehiculo/conductor de forma remota, etc, [1].

\section{TECNOLOGÍAS INALÁMBRICAS APLI- CADAS AL TRANSPORTE}

\subsection{Tipos de redes inalámbricas según su alcance}

Al definir algunos de los servicios que se pueden prestar haciendo uso de las comunicaciones inalámbricas entre vehículos, es necesaria la definición de las tecnologías actuales que hagan posible la implementación de estos, procurando eliminar o corregir los problemas que se puedan presentar, teniendo en cuenta que los tipos de redes existentes, fueron diseñadas para otro tipo de entornos, y aunque parece que funcionan de forma adecuada, para este tipo de servicios, pueden presentar problemas e incompatibilidades que se deben ir mejo- 
rando. Estos sistemas tienen que cumplir con unos requisitos mínimos que permita garantizar la comunicación en tiempo real, la privacidad de los datos, el enlace entre vehículos y las redes de acceso primarias entre otros. Como aporte inicial se describen los tipos de estructuras inalámbricas con base en su alcance:

PAN (Redes de área personal). Ofrece servicio a comunicaciones de carácter local, con un alcance máximo de 10 metros. Auriculares de teléfonos, dispositivos GPS inalámbricos, dispositivos de reproducción de música, identificación de señales de tráfico y dispositivos de comunicación instalados como parte de la electrónica del vehículo, son algunas de las aplicaciones disponibles. Dentro de las tecnologías disponibles para estas aplicaciones encuentran Bluetooth (IEEE 802.15), Redes Zigbee, RFID (Sistemas de identificación por radiofrecuencia).

WLAN (Red de área local inalámbrica). Permiten el intercambio de información dentro del vehículo y entre varios automóviles. No obstante, este tipo de redes no fue diseñada para movilidad sino para evitar el uso de cables en las oficinas. Los protocolos en los cuales se basan las WLAN, son los correspondientes a la IEEE 802.11, sin embargo existen estándares que soportan una comunicación V2V (entre automóviles) y V2I (entre autos y la infraestructura), para transmisión de información de seguridad, incidencias en carretera, etc [3].

WMAN (La red de área metropolitana inalámbrica). Cuenta con una cobertura de más o menos $50 \mathrm{~km}$ y soporta la integración de varios servicios como transmisión de voz, datos y video. IEEE 802.16 WIMAX, es usado para comunicaciones fijas en infraestructura y está en desarrollo el soporte para comunicaciones moviles, empleando la extensión del protocolo original IEEE 802.16e.

WAN (Wide Area Network). Da cobertura a un país o un continente. Las tecnologías disponibles en este escenario, son las basadas en los protocolos UMTS, GPRS y GSM. Estas redes son ideales para ser utilizada a nivel vehicular. Su mayor limitación se encuentra en los costes de la llamada, retardos de transmision y saturación de las células por el gran numero de usuarios [2].

\section{SISTEMAS DE COMUNICACIONES EX- TRA VEHICULARES}

\subsection{WLAN}

La WiFi (Wireless Fidelity) define la norma que garantiza la interoperabilidad de las denominadas redes de área local inalámbricas. Es un sistema flexible, muy utilizado como alternativa a las redes LAN cableadas o como extensión de estas [4].

IEEE 802.11 (ver tabla 1), define el protocolo para la implementación de dos tipos de redes:

Redes Ad-hoc. Compuesta únicamente por estaciones independientes dentro de una misma área de comunicación mutua a través del medio inalámbrico. Una WLAN adhoc se crea de manera espontánea. Basta con encender dos tarjetas de red para que exista comunicacion entre ellas. La principal distintiva de una Ad-hoc es su limitación temporal y espacial. Estas limitaciones obligan a que las operaciones de creación y disolución sean lo suficientemente sencillas y potentes como para permitir el uso de este tipo de redes a usuarios no experimentados.

Redes cliente / servidor. Utilizan un punto de acceso que controla la asignación del tiempo de transmision para todas las estaciones. Por tanto, deja de ser especificamente de contienda y cada estación pasa a tener el control del medio solo cuando es autorizado por el punto de acceso centralizado, ya sea entre la propia red sin cables o entre esa red y otras de tipo cableado.

Cualquiera de las dos anteriores configuraciones debe permitir la función de itine- 
rancia (roaming); es decir, que los usuarios puedan entrar y salir de las redes disponibles, ya sean Ad-hoc o de punto de acceso, de una forma totalmente transparente al usuario de la misma manera que ocurre en telefonía móvil.

Tabla 1. Tipos de WI-FI IEE 802.11

\begin{tabular}{|c|c|c|c|c|c|}
\hline Estándar & $\begin{array}{c}\text { Frecuencia de } \\
\text { operación }\end{array}$ & Rendimiento & $\begin{array}{c}\text { Tasa de envio } \\
\text { nominal }\end{array}$ & $\begin{array}{c}\text { Alcance } \\
\text { (interiores) }\end{array}$ & $\begin{array}{c}\text { Alcance } \\
\text { (esteriores) }\end{array}$ \\
\hline 802.11 & $2,4 \mathrm{GHZ}$ & 0,9 Mbps & 1.Mbps & $20 \mathrm{mts}$ & 100 mits \\
\hline $802.11 \mathrm{a}$ & $5 \mathrm{GHZ}$ & 2,33 Mbps & 2Mbops & $35 \mathrm{mts}$ & $120 \mathrm{mts}$ \\
\hline 802.116 & $2,4 \mathrm{GHZ}$ & $4,3 \mathrm{Mbps}$ & 11.Mbps & $38 \mathrm{mts}$ & $140 \mathrm{mts}$ \\
\hline $802.11 \mathrm{~g}$ & $2,4 \mathrm{GHZ}$ & 19Mbps & 54 Mbops & $38 \mathrm{mts}$ & $140 \mathrm{mts}$ \\
\hline $802.11 \mathrm{n}$ & $2,45 \mathrm{GHZ}$ & 144 Mbos & 300 Mbos & $70 \mathrm{mts}$ & $250 \mathrm{mts}$ \\
\hline
\end{tabular}

802.11p WAVE. El modelo de acceso inalámbrico en el entorno vehicular WAVE define una arquitectura y un conjunto de servicios e interfaces complementarios que habilitan comunicaciones inalámbricas seguras V2V y V2I, utilizando en espectro electromagnético de $5.9 \mathrm{GHz}$. Proporciona las bases para un amplio abanico de aplicaciones en el ámbito del transporte, incluyendo seguridad en vehículos, peajes automáticos, navegación mejorada, gestión de trafico, entre otros. La familia IEEE 1609 family of standards for wireless access in vehicular environments (WAVE) esta dividida en cuatro tipos de topologías: a) IEEE P1609.1, b) IEEE P1609, c) IEEE P1609.3, d) IEEE P1609.

WAVE constituye además el núcleo de los sistemas de comunicaciones dedicados de corto alcance DSRC.

DSRC (Dedicated short range communications). Incluyen transmision de datos de corto y medio alcance que soportan operaciones de seguridad publica y privada en entornos de comunicaciones de vehiculo a vehículo, vehículo a infraestructura y o viceversa. DSRC esta basado en la especificación IEEE 802.11p WAVE, proporcionan tasas de transferencia muy altas en circunstancias donde es importante minimizar los tiempos de latencia en el establecimiento de los canales y el aislamiento de zonas de comunicaciones relativamente pequeñas. Se usa en aplicaciones que involucran cobro electrónico de peajes con tarjeta de crédito o debito, solicitud o recepción de información del viajero y/o asistencia en ruta y automatización de información regulatoria entre coches pesados y estaciones de pesaje. Además es capaz de hacer envíos en modo broadcast o unicast, según las necesidades, integrando todos los receptores que se encuentran cercanos en una zona reducida de terreno en una misma red.

\subsection{WMAN}

WIMAX (interoperabilidad mundial para Acceso por Microondas), proporciona accesos concurrentes en áreas de hasta $48 \mathrm{~km}$ de radio a velocidades de hasta $70 \mathrm{Mbps}$, sin requerir visión directa con las estaciones base. Fue diseñado como solución de última milla en redes MAN, para prestar servicios a nivel comercial. [11]

El IEEE 802.16 con revisiones específicas se ocupa de dos modelos de uso:

Fijo. Llamado 802.16-2004 del IEEE (reemplaza versiones del IEEE 802.16a y 802.16d). Es una opción inalámbrica para acceso a Internet de banda ancha que provee una solución de clase interoperable que funciona en $2.5 \mathrm{GHz}$ (autorizado), $3.5 \mathrm{GHz}$ y $5.8 \mathrm{GHz}$ (exento de licencia). Provee una alternativa inalámbrica a las líneas digitales de suscriptor de cualquier tipo (xDSL) [12]. Usa OFDM (multiplexacióm por división de frecuencia ortogonal) para la optimización de servicios inalámbricos, con varias portadoras llevando datos en paralelo. En este caso, la señal OFDM esta dividida en 256 canales en lugar de 64 como ocurre con el estándar 802.11.

Móvil. El 802.16e del IEEE es una revisión para la especificación base 802.16-2004 que añade portabilidad y capacidad para clientes moviles. Se soporta en OFDMA (acceso múltiple por división ortogonal de frecuencia) que es una técnica similar a OFDM, 
no obstante, va un paso más allá agrupando subportadoras múltiples en subcanales. Específicamente, una sola estación cliente del suscriptor podría usar todos los subcanales dentro del periodo de la transmision, o múltiples clientes podrían transmitir simultáneamente usando cada uno una porción del número total de subcanales.

\subsection{Comparación entre WIFI y WIMAX}

Las tablas 2 y 3 muestran las características más relevantes de cada tecnología y en la tabla 4 se establece una comparativa entre ellas a nivel de alcance, cobertura, escalabilidad [13].

Tabla 2. Estándar WIFI.

\begin{tabular}{|c|l|}
\hline Característica & \multicolumn{1}{|c|}{ WIFI 802.11 } \\
\hline Alcance & $\begin{array}{l}\text { Optimizado para usuarios en un radio de 100m. } \\
\text { Aumento de alcance con antenas de gran ganancia } \\
\text { y amplificadores en los puntos de acceso. }\end{array}$ \\
\hline Cobertura & Optimizada para entornos interiores. \\
\hline Escalabilidad & El ancho de banda del canal es fijo a 20 MHZ. \\
\hline $\begin{array}{c}\text { Velocidad de } \\
\text { transferencia }\end{array}$ & 300 Mbps con 2 canales de 20 Mhz \\
\hline
\end{tabular}

Tabla 3. Estándar WIMAX.

\begin{tabular}{|c|l|}
\hline Característica & \multicolumn{1}{|c|}{ WIMAX 802.16 } \\
\hline Alcance & $\begin{array}{l}\text { Optimizado para un tamaño típico de célula de } \\
7-10 \mathrm{Km} \text { de alcance. }\end{array}$ \\
\hline Cobertura & Optimizada para entornos exteriores. \\
\hline Escalabilidad & $\begin{array}{l}\text { Ancho de banda flexible de 1.5 a 20 MHZ para } \\
\text { bandas con/sin licencia. } \\
\text { Reutilización de la frecuencia. } \\
\text { Planificación de las células para proveedores de } \\
\text { servicios. }\end{array}$ \\
\hline $\begin{array}{l}\text { Velocidad de } \\
\text { transferencia }\end{array}$ & Hasta 75 Mbps en canales de 20 Mhz \\
\hline
\end{tabular}

Tabla 4. Comparación WIMAX.

\begin{tabular}{|c|l|}
\hline Característica & \multicolumn{1}{|c|}{ Explicación técnica } \\
\hline Alcance & $\begin{array}{l}\text { WIMAX tolera 10 veces } \\
\text { más retardo multicamino } \\
\text { que } 802.1 .\end{array}$ \\
\hline Cobertura & $\begin{array}{l}\text { Modulación adaptativa } \\
\text { 256 canales en WIMAX } \\
\text { frente a 64 de WIFI. }\end{array}$ \\
\hline Escalabilidad & $\begin{array}{l}\text { El número de canales } \\
\text { de WIMAX solo está } \\
\text { limitado por el espectro } \\
\text { disponible. }\end{array}$ \\
\hline
\end{tabular}

\subsection{Sistemas móviles celulares}

GSM (Sistema móvil global). Según [22], a comienzos de los años ochenta, muchos países en Europa habían desarrollado su propio sistema de telefonía celular análoga que impedía la interoperabilidad más allá de las fronteras de cada país. En 1982, el CEPT (Conference of European post and telecommunications) creó un grupo de trabajo para desarrollar un sistema inalámbrico móvil paneuropeo al que denomino Groupe Speciale Mobile que incluyera las siguientes funcionalidades: roaming Internacional, soporte para la introducción de nuevos servicios, eficiencia espectral y compatibilidad con la RDSI. En 1989, la responsabilidad por el desarrollo de GSM fue transferida al ETSI (European telecommunications standards institute) que denomino al proyecto como Global System for Mobile Communications.

GSM es un sistema de conmutación de circuitos, diseñado originalmente para voz, al que posteriormente se le adicionaron algunos servicios de datos: servicio de mensajes cortos, un servicio de entrega de mensajes de texto de hasta 160 caracteres y un servicio de datos GSM, que permite una tasa de transferencia de $9.6 \mathrm{kbps}$.

GPRS (General packet radio system). Es una tecnología de generación 2.5, es decir, representa un estado de transición entre la segunda y tercera generación. Provee acceso de radio paquetes sobre GSM existente; en este sentido, constituye una extensión de conmutación de paquetes sobre dicha red. GPRS acomoda más eficientemente fuentes de datos que tienen por lo general una naturaleza a ráfagas.

UMTS (Universal mobile telecommunications system). Existe, [22] dentro del ITU, un grupo estratégico denominado Internacional mobile telecommunications, IMT- 2000, que tiene como objetivo de trabajo definir las interfaces entre las redes de tercera generación y las que evolu- 
cionaron a partir de GSM por una parte, y desde ANSI-41 (su contraparte americana), por otra, para permitir la itinerancia entre estas estructuras. Por el lado de GSM, el ETSI-European Telecommunications Standards Institute y un grupo de organismos asociados decidieron, en el año de 1998, emprender un proyecto denominado 3GPP (Third generation partnership project) que buscaba establecer los parámetros para un sistema móvil de tercera generacion que tuviera una red núcleo basada en la evolución de GSM y cuya red de acceso estuviera basada en todas las tecnologías de radio acceso (FDD y TDD). El 3GPP empezó a denominar a los sistemas moviles de tercera generacion como Servicio universal de telecomunicaciones moviles (UMTS). Ha sido presentada como la culminación de la convergencia de Internet y las redes moviles, en ella, los usuarios tendrán la posibilidad de acceder a contenidos y servicios multimedia de banda ancha independientemente del lugar donde se encuentren.

\subsection{Comparación entre GSM, GPRS y UMTS}

En la tabla 5 se comparan los parámetros mas relevantes de las tecnologías de telefonía móvil celular.

Tabla 5. Paralelo entre GSM, GPRS y UMTS.

\begin{tabular}{|c|c|c|c|}
\hline Caracteristica & \multicolumn{3}{|c|}{ Tecnologías celulares } \\
\hline $\begin{array}{c}\text { Velocidad de } \\
\text { transmisión (Kbps) }\end{array}$ & GSM & GPRS & UMTS \\
\hline $\begin{array}{c}\text { Velocidad de } \\
\text { transmisión (Kbps) }\end{array}$ & $9,6 \mathrm{Kbps}$ & $\begin{array}{c}171,2 \text { kbit s teóricos, } \\
\text { pero en la práctica } \\
\text { es de } 40 \text { Kbps de } \\
\text { bajada y } 9,6 \mathrm{Kbps} \\
\text { de Subida. }\end{array}$ & $\begin{array}{c}144 \text { (rural exteriores) } \\
384 \text { (urbano exteriores) } \\
\text { Y hasta } 2 \text { Mbps en } \\
\text { interiores y exteriores de } \\
\text { bajo rango de distancias. }\end{array}$ \\
\hline Tipo de Servicio & $\begin{array}{l}\text { Voz y SMS } \\
\text { (Short } \\
\text { Messsage } \\
\text { Services) } \\
\end{array}$ & $\begin{array}{l}\text { Vos, Datos, } \\
\text { Servicios IP }\end{array}$ & Voz y Datos \\
\hline Protocolo MAC & $\begin{array}{l}\text { FDMA, TDMA } \\
\text { y CDMA }\end{array}$ & TDMA & \\
\hline Modulación PHY & $\begin{array}{c}\text { GMSK } \\
\text { (Gaussian } \\
\text { Minimum Shift } \\
\text { Keying) }\end{array}$ & $\begin{array}{l}\text { GMSK (Gaussian } \\
\text { Minimum Shift } \\
\text { Keying) }\end{array}$ & $\begin{array}{l}\text { W-CDMA (Acceso } \\
\text { múltiple por división de } \\
\text { codigo de banda de } \\
\text { ancha). }\end{array}$ \\
\hline Bandas de Frecuencia & $\begin{array}{c}900 \mathrm{Mhz} \text { y } 1800 \\
\mathrm{Mhz}\end{array}$ & $\begin{array}{c}900 \mathrm{Mhz} \text { y } 1800 \\
\text { Mhz }\end{array}$ & $\begin{array}{c}1885 \text { a } 2025 \mathrm{Mhz} \text { y } 2110 \\
\text { a } 2200 \mathrm{Mhz} \text {. }\end{array}$ \\
\hline
\end{tabular}

\section{SISTEMAS DE COMUNICACIONES IN- TRAVEHICULARES}

Las comunicaciones intravehiculares permiten el intercambio de información den- tro del vehiculo. Existen dos tipos, las cableadas, que transmiten datos entre todos los componentes encargados de la gestión de la conducción, seguridad y sistemas internos del automóvil. Normalmente, los buses de comunicaciones cableados son multiplexados y se basan en tecnología CAN (Controller area network) de Bosch, el estándar de facto en automoción. El otro tipo de sistemas de comunicaciones intravehiculares están basados en redes inalámbricos, que a continuación se describe.

Dada la menor fiabilidad de este tipo de redes, fundamentalmente en lo referente a tiempo real e interferencias, esta dedicado única y exclusivamente a dar servicio a sistemas de confort y multimedia, dejando de lado temas relacionados con seguridad. El manos libres del teléfono móvil, navegadores, musica o video son algunas de las aplicaciones más comunes. No obstante, la aplicación de este tipo de redes tiene una trascendencia incalculable en el futuro de la automoción. De la misma manera en que gran parte de la electricidad y el cableado de los vehículos fueron sustituidos por buses de comunicaciones para, entre otras cosas, ahorrar peso y costes, en un futuro posiblemente cercano, esos buses de datos serán sustituidos por redes inalámbricas, eliminando definitivamente la mayoría del cableado de los carros. De hecho, se estima que por termino medio en un vehiculo se encuentran instalados 1900 cables, que vienen a ser unos $2 \mathrm{~km}$. de cableado y representan un peso de $40 \mathrm{~kg}$. De otro lado las rutas de los cables deben ser variadas en cada ciclo de producción, representando un coste añadido. Por todo esto, la integración de sensores inalámbricos dentro del vehiculo, supone una interesante alternativa a la falta de flexibilidad de las redes cableadas, habilitando una malla interconectada inalámbrica para gestionar toda la información dentro del vehiculo. En consecuencia, toda actividad en el area de inalámbrico intravehiculares supone una oportunidad de negocio para futuros desarrollos. 
Dentro de las aplicaciones que pueden ser utilizadas en este tipo de sistemas esta:

- Bluetooth.

- Zigbee.

- UWB.

También se incluye en esta clasificación a los RFID pasivos [8], que forman parte de sistemas de seguridad como la identificación de la llave del auto o de la tarjeta [9].

Bluetooth. Especificación industrial para las WPAN que facilita el transporte de datos entre diferentes dispositivos mediante un enlace por radiofrecuencia en la banda de $2.4 \mathrm{GHz}$. Ha sido diseñado para permitir el desarrollo de dispositivos de comunicaciones de bajo coste, con bajo consumo $\mathrm{y}$ de corto alcance (1 metro, 10 metros, 100 metros). Utiliza Frequency hop-ping spread spectrum (FHSS) para la transmisión de datos, utilizando para ello 79 canales en la banda de los 2.4 GHZ para ISM (banda de frecuencia industrial, científica y medica) y así, contrarrestar las interferencias y la perdida de intensidad esporádica [5]. El funcionamiento habitual de las redes Bluetooth se rige por el esquema maestroesclavo. Uno de los dispositivos, denominado maestro, proporciona los valores de referencia de la conexión (como por ejemplo, la sincronización con su reloj y la secuencia de salto de frecuencia). Los demás puntos de conexión (hasta 7) reciben el nombre de esclavos e intercambian tráfico con el maestro. Esta red formada por ocho dispositivos de corto alcance no es más que una PAN y se denomina piconet.

Una de las características fundamentales Bluetooth es que el flujo de paquetes puede circular entre el maestro y cualquier otro elemento de red; no obstante, los diferentes componentes pueden cambiar sus roles entre si y, de esta forma, un maestro puede transformarse en esclavo y viceversa, dependiendo de la necesidades de las aplicaciones que soporten las comunicaciones. Además, los nodos son autónomos, es decir, que no estan asociados a ordenadores, sino que pueden ser simples sensores o actuadores independientes y con alimentación autónoma, formando una WSAN (Wireless sensor and actuator network). La especificación Bluetooth permite también la interconexión de dos o más piconets, formando así una scatternet, en la que alguno de los esclavos ejerce de puerta de enlace de entre dos topologías, siendo maestro en una y esclavo en otra. En la actualidad, las comunicaciones Bluetooth son ampliamente utilizadas en el ambito automovilístico. En la actualidad se pueden encontrar multitud de aplicaciones y sistemas que emplean conexiones entre diferentes dispositivos del vehículo con una PDA, con el teléfono móvil, con un reproductor MP3 o con un GPS.

Zigbee. Se basan en el estándar IEEE 802.15.4-2003. Otras redes existentes de similares especificaciones son WirelessHART, ISA100, 6lowpan. Son totalmente autónomas y cuentan con alimentación propia, con lo que es posible acoplarlos a cualquier tipo de sistema de forma remota (como sensores o actuadores), sin necesidad de funcionar sobre un ordenador. La red en su conjunto utilizara una cantidad muy pequeña de energía de forma que cada dispositivo individual puede tener una autonomía de hasta 5 años antes de necesitar un cambio en su sistema de alimentación. No obstante el de bajo consumo de la red, limita el alcance de cada unidad a un valor muy reducido. Sin embargo, la topología como tal puede aumentar ilimitadamente su cobertura mediante configuraciones en malla autoorganizativa, que hace que todos los nodos se comporten como routers $y$, de esta manera dar servicio a medianas extensiones.

Funciona en la bandas $2.4 \mathrm{GHz}, 915 \mathrm{MHz}$ (en Norteamérica) y $868 \mathrm{MHz}$ (en Europa). Puede soporta comunicaciones en tiempo real, utilizando conceptos muy similares a las redes tipo CAN. Para ello incluye la posibilidad de definir el nivel de prioridad de los mensajes que son emitidos por los nodos. 
La principal ventaja y posibilidad de interacción de las redes Zigbee son la de integrar su sistema de comunicaciones inalámbrico con sensores y actuadores para que funcionen de manera totalmente autónoma, sin necesidad de cableado, además su robustez incluye auto reconfiguración de la red en caso de nodos estropeados. Todos estos aspectos hacen que sus aplicaciones en el campo de la automoción sean multiples, sobre todo a la hora de sustituir buses cableados cuyos nodos exigen tiempo real.

UWB (Ultra wide band). La tecnología UWB puede utilizarse para transmitir voz, video u otro tipo de datos digitales. Su principal ventaja respecto a otras, es que puede transmitir mas datos utilizando menos potencia que el resto de sistemas disponibles. Adicionalmente, los equipos de radio necesitan menos componentes, por lo que se convierte en una solución económica.

De acuerdo a la FCC, los dispositivos UWB deben funcionar con su ancho de banda a -10 dB en el interior del margen de frecuencias que se extiende desde 3,1 hasta 10,6 $\mathrm{GHz}$ y con una densidad espectral de potencia máxima de emisión de -41,3 dBm/MHz [6]. Debido a esta limitación de potencia, el alcance de estos sistemas es bastante reducido. No obstante, esto se convierte en una ventaja cuando se desea combinar varios radio-enlaces en un espacio relativamente pequeño, como por ejemplo una oficina o un apartamento.

El funcionamiento de UWB se basa en transmitir secuencias de pulsos muy estrechos, donde estos se sitúan de forma precisa en el tiempo (incluye desviaciones inferiores al nanosegundo). La modulación en Zigbee consiste en variar la posición de los pulsos empleando códigos PN (técnica de espectro ensanchado), obteniendo como resultado un espectro de banda ancha. Adicionalmente, dado que las señales UWB son de baja potencia, causan muy poca interfe- rencia al resto de señales. Por ejemplo, estudios demuestran que la interferencia de UWB sobre los sistemas GPS es menor a las causadas por diversos equipos eléctricos como un secador de pelo, un taladro o una fuente de alimentación de PC [7].

En comparación con otro tipo especificaciones inalambricas, como WIFI WIMAX; UWB proporciona una mayor velocidad con una gran eficiencia en potencia, lo que permite el desarrollo de dispositivos portátiles de gran autonomía. Su alcance es similar a Bluetooth, debido principalmente a las limitaciones de potencia impuestas. Eliminando estas restricciones, el alcance de UWB se estima que podría ser similar o incluso superior al proporcionado por las tecnologías 802.11. El principal campo de aplicación se orienta hacia la electrónica del hogar, en la interconexión de periféricos tales como impresoras o monitores con el PC, o en la distribución de señales HDTV a distintos receptores de TV. En el campo de la automoción, es de especial interés en los sistemas de seguridad inteligentes como aviso de colisión, detección de peatones o de emergencia. [10]

En las tabla 6 y 7 se muestran los aspectos más importantes de cada técnica.

Tabla 6. Comparación entre redes intra-vehiculares (RFID, Bluetooth)

\begin{tabular}{|l|c|c|}
\hline \multirow{2}{*}{ Nombre } & \multicolumn{2}{c|}{ Tecnología } \\
\cline { 2 - 3 } & RFID (pasivo) & Bluetooth \\
\hline $\begin{array}{l}\text { Velocidad de transmisión } \\
\text { (kbps) }\end{array}$ & 28 & 720 \\
\hline Alcance (m) & $0.1-10$ & $1-10$ \\
\hline Tamaño de la red (nodos) & 1000 & 7 \\
\hline Tiempo de inicialización & Decenas de mseg & Seg \\
\hline Topología de red & Estrella & Estrella \\
\hline Protocolo MAC & Q & TDMA \\
\hline Modulación PHY & ASK & FHSS \\
\hline Requisitos de memoria & 4 KB & $250 \mathrm{~KB}$ \\
\hline Potencia en transmisión & 0 & 1 mW \\
\hline Aplicaciones & Sistema de gestión & $\begin{array}{l}\text { Reemplazo de } \\
\text { cables }\end{array}$ \\
\hline
\end{tabular}


Tabla 7. Comparación entre redes intra-vehiculares (ZigBee, UWB)

\begin{tabular}{|c|c|c|}
\hline \multirow{2}{*}{ Nombre } & \multicolumn{2}{|c|}{ Tecnología } \\
\hline & ZigBee & UWB \\
\hline $\begin{array}{l}\text { Velocidad de trans- } \\
\text { misión (kbps) }\end{array}$ & $20-250$ & $20-250$ \\
\hline Alcance (m) & $1-100$ & $1-100$ \\
\hline $\begin{array}{l}\text { Tamaño de la red } \\
\text { (nodos) }\end{array}$ & $256 / 65536$ & $256 / 65536$ \\
\hline $\begin{array}{l}\text { Tiempo de inicial- } \\
\text { ización }\end{array}$ & mseg & $<<1$ seg \\
\hline Topología de red & $\begin{array}{l}\text { Flexible (estrella, } \\
\text { malla, hibrida) }\end{array}$ & $\begin{array}{l}\text { Flexible (estrella, } \\
\text { malla, hibrida) }\end{array}$ \\
\hline Protocolo MAC & $\begin{array}{l}\text { CSMA/CA y } \\
\text { TDMA }\end{array}$ & $\begin{array}{c}\text { CSMA/CA y } \\
\text { TDMA }\end{array}$ \\
\hline Modulación PHY & DSSS & DS-UWB \\
\hline $\begin{array}{l}\text { Requisitos de } \\
\text { memoria }\end{array}$ & $4-32 \mathrm{~KB}$ & $4-32 \mathrm{~KB}$ \\
\hline $\begin{array}{l}\text { Potencia en trans- } \\
\text { misión }\end{array}$ & $<1 \mathrm{~mW}$ & $1 \mathrm{~mW} / \mathrm{Mbps}$ \\
\hline Aplicaciones & $\begin{array}{l}\text { Monitorización y } \\
\text { control }\end{array}$ & $\begin{array}{l}\text { Localización de } \\
\text { alta precisión, } \\
\text { multimedia }\end{array}$ \\
\hline
\end{tabular}

\section{ARQUITECTURAS}

CALM (Communications, air interface, long and medium range). Es una arquitectura referente de ISO para dar soporte a comunicaciones en entornos móviles, más específicamente, en aquellos relacionados con tecnologías inteligentes de transporte, soporta comunicaciones continuas a través de distintos interfaces y medios fisicos como los IEEE 802.11, 802.11p, 802.15, 802.16e, 802.20, telefonía móvil 2G/3G/4G. Dentro de las aplicaciones fundamentales se incluye el soporte a servicios de Internet en entornos móviles, y soporte a la nueva generación de aplicaciones ITS (protección vehicular) [14].

Los agentes que se involucran en los diferentes servicios son los OBU (On Board Units) implementados en automóviles, y los RSU (Road Side Unit) que son los entornos instalados en la infraestructura. Los principales servicios ofrecidos se enfocan a operaciones con vehículos comerciales (CVO), gestión de cobros (payment), información del tráfico y seguridad en comunicaciones vehiculares (VSC).
La contribución trascendente de la arquitectura CALM es que por primera vez todos los actores que participan en el ambito del transporte (compañías automovilísticas, compañías de construcción de infraestructuras, agentes sociales, administración, etc.) han colaborado en la construcción de un esquema que integra todos los ámbitos de comunicaciones en el transporte [16]. La Fig. 1 muestra la distribución de las interfaces de acceso al entorno usadas en CALM.

CVIS (Cooperative Vehicle- Infrastructure Systems. Se derivada de la CALM, de la que usa su soporte fisico y la estructura general. Su mayor aporte es la implementación de los diferentes servicios y aplicaciones vehículo-infraestructura y vehículo-vehículo [21]:

CVIS (Cooperative vehicle - infrastructure systems). Se derivada de la CALM, de la que usa su soporte fisico y la estructura general. Su mayor aporte es la implementación de los diferentes servicios y aplicaciones vehículo-infraestructura y vehículovehículo [21]:

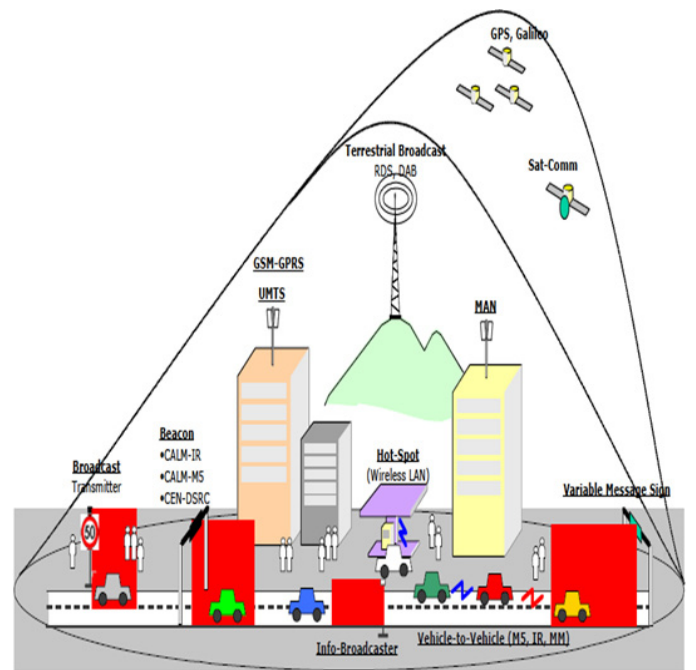

Fig. 1. Distribución de interfaces de acceso al entorno, utilizados en la arquitectura CALM. Fuente ISO [20].

Dentro de los servicios que utilizan comunicaciones vehículo-infraestructura estan [16]: 
- Aviso de ángulo muerto.

- Aviso de velocidad en curvas.

- Aviso de ambulancia.

- Aviso de paso de paso a nivel, interseccion.

- Aviso de semáforo en ambar.

- Aviso de paso de tunel bajo.

- Aviso de cruce de peatones.

- Aviso de condiciones de la carretera (hielo, lluvia).

- Aviso de obras en la via.

Igualmente, las prestaciones vehículo-vehículo incluyen: [18]:

- Aviso de automóvil con luces de emergencia.

- Aviso de zona angulo muerto.

- Aviso de colisión.

- Aviso de cambio de carril.

- Aviso de llamada de emergencia.

- Aviso de automóvil en sentido contrario.



Fig. 2. Medios de comunicación CALM implementados por CVIS [19].

VII (Vehicle infrastructure integration). El programa Americano de integración vehículo-infraestructura, trata de desarrollar sistemas de comunicaciones que soporten el intercambio de datos entre todos los actores que intervienen en la seguridad vial a fin de reducir el numero de accidentes y mejorar la eficiencia de las redes de transporte. Los objetivos básicos de este programa son muy similares a los que se pretenden conseguir en Europa a través de la implantación de la arquitectura CALM. La iniciativa tiene tres prioridades [15]:

- Evaluación del modelo de negocio y aceptación de los actores involucrados.

- Validación de la tecnología, en particular de los sistemas de comunicaciones.

- Desarrollo de las estructuras legales necesarias para que los sistemas sean viables a largo plazo.

Dentro de las aplicaciones soportadas por el programa VII [19] se pueden nombrar:

- Aviso de violación de señal de tráfico.

- Gestión de corredores viarios.

- Aviso de violación de señal de Stop.

- Información al conductor.

- Aviso de velocidad excesiva en curva.

- Pago electrónico.

- Luces de freno electrónicas.

- Información de meteorológica local.

- Alerta temprana de incidencias los conductores.

- Operaciones de mantenimiento invernal.

- Señalización dentro del vehículo.

- Generación de información cartográfica

- Medición de acceso a autopistas.

- Ajuste y temporización de señales de tráfico.

VANET (Vehicular Ad-Hoc network). Se trata de una red ad-hoc donde sus nodos corresponden automóviles, camiones, autobuses, etc. Por lo tanto, contiene la posibilidad de que los dispositivos formen la red en pleno movimiento (por ejemplo mientras se circula por una autopista). En cuanto a nomenclatura, VANET es de tipo de MANET (Mobile Ad-Hoc Network), es decir, una red ad-hoc móvil, aunque se aclara que MANET describe sobre todo un cam- 
po de investigación académico, mientras que el termino VANET esta más enfocada a una aplicación.

Actualmente esta en pleno desarrollo e investigación. De hecho, existen varios grupos de trabajo, tanto por parte de las universidades y los gobiernos, como de la industria, que trabajan en este campo debido a la multitud de posibles aplicaciones que podría suponer su utilización. Algunos de los consorcios son por ejemplo el Vehicle Safety Communications (VSC EEUU), Car to Car Communication Consortium (C2CCC Europa), Internet ITS (Japón), Sigmobile (EEUU) y el propio IEEE. La Fig. 3 muestra esquemáticamente como funciona la red Ad-hoc vehicular.

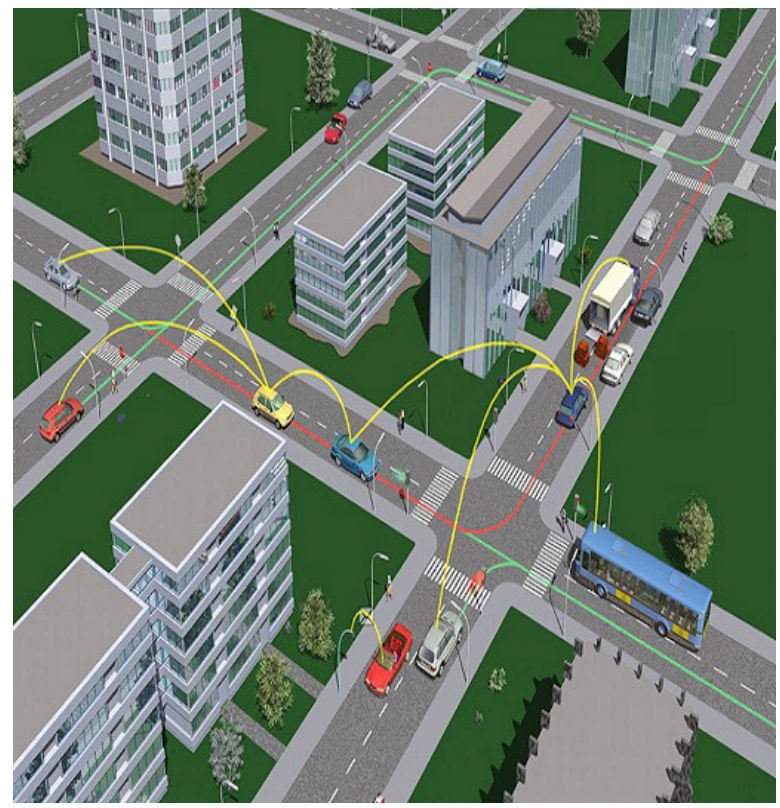

Fig. 3. Ejemplo de funcionamiento de VANET [21].

Referencias Bibliográficas

[1] T. Vaa. M. Penttinen, I. Spyropoulou, Intelligent transport systems and effects on road traffic accidents: state of the art, , IEEE, mayo 2007.

[2] M. Xiaomin, C. Xianbo, Delay and broadcast reception rates of highway

\section{CONCLUSIONES}

Tomando como referencia el consumo eléctrico, se tiene que ZigBee tiene un consumo de $30 \mathrm{~mA}$ en transmisión, siendo menor al Bluetooth con $40 \mathrm{~mA}$, debido a que ZigBee en el momento en el que no hay tráfico cambia a un estado "dormido".

Desde el punto de vista de velocidad, ZigBee cuenta con una rata de transmisión de $250 \mathrm{kbps}$, frente a Bluetooth que logra $1 \mathrm{Mbps}$, lo que le permite a esta última su utilización en teléfonos móviles y periféricos de vehículos, contrario a ZigBee que en este tipo de entornos no tendría cabida.

RFID por sus característica de latencia baja y debido a la capacidad mínima de transporte información, se utiliza en sistemas de seguridad como llave o tarjetas de acceso al vehículo, y tele-peaje o identificación del vehículo.

La tecnología UWB en la actualidad es la menos utilizada, encontrándose aun en auge, pero debido a sus cualidades relacionadas con la potencia de transmisión, en el futuro puede remplazar al Bluetooth en aplicaciones que requiera gran ancho de banda.

En cuanto a las arquitecturas de telefonía celular, UMTS, ofrece los mayores anchos de banda, ofreciendo una amplia gama de servicios, convirtiéndola en una de las mejores opciones para la implementación de redes vehiculares de última generación. safety applications in vehicular Ad Hoc networks, , IEEE, septiembre 2007.

[3] D. Sanjeev, Analogy of promising wireless technologies on different frequencies: Bluetooth, WiFi, and Wi- 
MAX, IEEE, Junio 2007.

[4] J. Naranjo, F. Jiménez, J. Armingol, Entornos inteligentes basados en redes inalámbricas: aplicaciones al transporte, automóvil inteligente/conectado y seguridad vial, Dic 2008.

[5] McDermott-Wells, P, IEEE, What is Bluetooth?, Diciembre 2004.

[6] X. Bin, X. Hong, L. Qian, X. Nan, Linear combination method for UWB vehicular radar pulse design, IEEE, Abril 2010.

[7] L. Yong-Taek, L. Kai, R. Frye, K. Hyun-Tai K. Gwang, Ultra-WideBand (UWB) Band-Pass-Filter using integrated passive device (IPD) technology for wireless applications, IEEE, Junio 2009.

[8] L. Donggeon, K. Seongyun, K Howon, P. Namje, Mobile platform for networked RFID applications, IEEE, Abril 2010.

[9] A. Masud, M. Mondal, K. Ahmed, Vehicular communication system for vehicle safety using RFID, IEEE, Abril 2010.

[10] M. Thakur, T. Quinlan, A. Ahmad, D. Hunter, Triple-

[11] format, UWB-WiFi-WiMax, radiooverfFiber Co-existence demonstration featuring low-cost 1308/1564 nm VCSELs and a reflective electroabsorption transceiver, IEEE, Mayo 2009.

[12] N. Begam, F. Rahman , K. Ahmed, Analysis of propagation model performance in WIMAX (IEEE 02.16e)-based wireless mobile vehicular networks, IEEE, Abril 2010.

[13] C. Jiann-Liang, C. Yao-Chung, L. YuShan, D. Hsien, Embedded WIMAXbased vehicular router for telematics computing, IEEE, Abril 2009.
[14] C. Chien-Ming, L. Chen-Yuan, C. Wei-Min, L. Kun-chan, A feasibility study on vehicle-to-infrastructure communication WIFI vs. WIMAX, IEEE, junio 2009.

[15] A. Bohm , K. Lidstrom, M. Jonsson, T. Larsson, Evaluating CALM M5-based vehicle-to-vehicle communication in various road settings through field trials, IEEE, Abril 2010.

[16] J. Yao,Y. Xiaoguang, D. Wu, Urban traffic control tentative exploration in vehicle-infrastructure integration environment, IEEE, Abril 2010.

[17] T. Ernst, V. Nebehaj, R. Srase, CVIS: CALM proof of concept preliminary results, IEEE, Abril 2010.

[18] G. Grau, D. Pusceddu, S. Rea, O.Brickley, M. Koubek, Pesch D, Vehicle-2-vehicle communication channel evaluation using the CVIS platform, IEEE, Abril 2010.

[19] S. Pechberti, D. Gruyer, D. Gingras, F. Dupin, Design of a modular demonstrator for safety application systems the CVIS project, IEEE, Abril 2010.

[20] Communications Access for Land Mobiles, [en línea]. Consultada en Abril 10 de 2010, disponible en: http://www. isotc204wg16.org/pubdocs/The $\% 20$ CALM\%20Handbookv6-070301.pdf

[21] CVIS and ISO - CALM, [en línea]. Consultada en Febrero 20 de 2010, disponible en: http://www.cvisproject.org/download/Presentations/ Aalborg07/SS01/ SS01_Schalk\%20 as $\% 20$ speaker.pdf.

[22] [en línea]. Consultada en Enero 12 de 2011, disponible en neo.lcc.uma.es/ staff/jamal/portal/?q=content/vanetvehicular-adhoc- networks.

[23] A. Pachon, Evolución de los sistemas móviles celulares GSM, Octubre 2004. 